\title{
The Use of Aspirin to Reduce the Risk of Thrombotic Events in Patients With End-Stage Renal Disease: Protocol for a Randomized Controlled Trial
}

Tiago Lemos Cerqueira ${ }^{1,2,3^{*}}$, MPH, MD; Armando Fartolino Guerrero ${ }^{2,4}$, MD; Clara Krystal Pérez Fermin ${ }^{2,5}$, MD; Ricardo Wang ${ }^{2,6}$, MD; Evelin Elfriede Balbino ${ }^{2,7}$, PharmD; Janis L Breeze ${ }^{8,9}$, MPH, PhD; Paola Gonzalez Mego ${ }^{2,10}$, MD; Daniele Argentina Silva ${ }^{2,11}$, MD; Walid Ezzeldin $\mathrm{Omer}^{2,12}$, MD; Nathalie Monique Vandevelde ${ }^{2,13^{*}}$, PharmD, $\mathrm{PhD}$

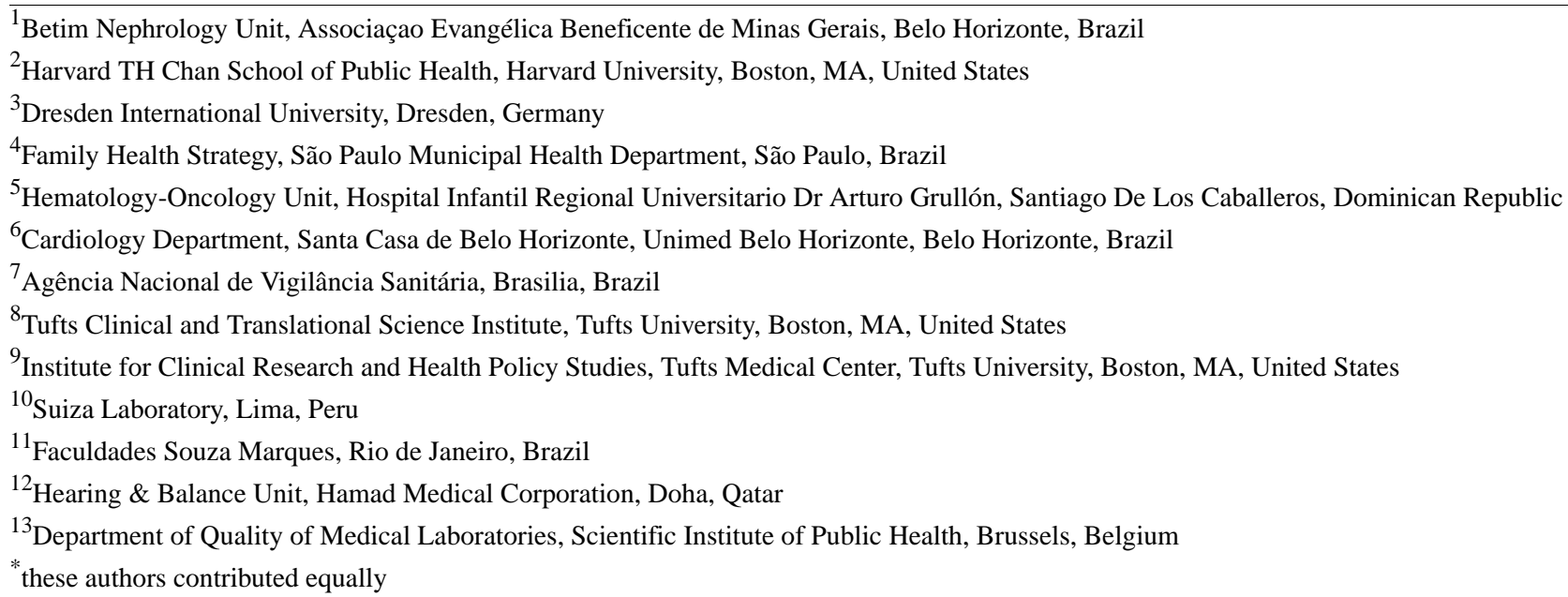

\section{Corresponding Author:}

Nathalie Monique Vandevelde, PharmD, PhD

Department of Quality of Medical Laboratories

Scientific Institute of Public Health

Rue Juliette Wytsmanstraat 14

Brussels,

Belgium

Phone: 32026425589

Email: nathalie.vandevelde@wiv-isp.be

\begin{abstract}
Background: End-stage renal disease (ESRD) is the last stage of chronic kidney disease, mainly caused by type 2 diabetes mellitus and characterized by an increased mortality risk related to cardiovascular disease. Low-dose aspirin (acetylsalicylic acid or ASA) seems to effectively prevent cardiovascular events in patients with ESRD. However, the number of interventional studies in this population remains limited and the mechanisms of aspirin-related bleeding remain poorly understood. Aspirin's efficacy and safety may be modified by the presence of type 2 diabetes mellitus or platelet hyperreactivity.

Objective: The overall objective of this protocol is to (1) evaluate aspirin's safety and efficacy in reducing the risk of thrombotic events in patients with ESRD on hemodialysis and (2) examine whether aspirin's efficacy is modified by the presence of type 2 diabetes mellitus or platelet hyperreactivity. Specifically, the primary objective is to compare the 12-month rate of any thrombotic event (cardiac death, nonfatal myocardial infarction, nonfatal stroke, arteriovenous fistula thrombosis) and Thrombolysis in Myocardial Infarction (TIMI) major bleeding in patients treated with aspirin compared to those on placebo. Secondary objectives are to test for effect modification of treatment by the presence of type 2 diabetes mellitus or platelet hyperreactivity and compare the rate of TIMI minor bleeding between treatment groups.
\end{abstract}

Methods: We developed a protocol for a phase 2 randomized, single-center, placebo-controlled, triple-blind, superiority clinical trial to assess the prophylactic efficacy and safety of aspirin in patients with ESRD and on hemodialysis. It follows the ethical 
principles of the Declaration of Helsinki of the World Medical Association. A total of 342 participants would be enrolled over 12 months at a large dialysis center. Patients will be randomized in a 1:1 ratio and stratified by presence of type 2 diabetes mellitus and platelet hyperreactivity to receive either oral aspirin $(100 \mathrm{mg} / \mathrm{d})$ or placebo for a treatment period of $12 \mathrm{months}$. An intention-to-treat statistical analysis will be performed.

Results: The randomized clinical trial will be performed after approval by the ethical committee of the participating center and registration at ClinicalTrials.gov.

Conclusions: We provide a protocol for a randomized controlled trial to evaluate the safety and efficacy of treatment with aspirin to reduce the risk of thrombotic events. In addition, such a study would further our understanding of the mechanism of aspirin-related bleeding and help identify subgroups of best-responders and patients with a higher risk of adverse events.

Registered Report Identifier: RR1-10.2196/10516

(JMIR Res Protoc 2018;7(8):e10516) doi: 10.2196/10516

\section{KEYWORDS}

randomized controlled trial; end stage renal disease; kidney failure, chronic; aspirin; prophylaxis; thrombosis; drug safety; bleeding; platelet activation; diabetes mellitus, type 2

\section{Protocol}

\section{Introduction}

\section{Background and Rationale}

Chronic kidney disease (CKD) is a pathology defined by a decrease in glomerular filtration rate (GFR) below 60 $\mathrm{mL} / \mathrm{min} / 1.73 \mathrm{~m}^{2}$ or by the presence of kidney damage for at least 3 months [1]. It affects 200 million people worldwide, and the main risk factor for this disease is diabetes mellitus [2-4]. The life expectancy of CKD patients decreases with the severity of kidney impairment [5]. The most severe stage is end-stage renal disease (ESRD) and refers to patients undergoing renal replacement therapy (RRT) or for whom the GFR is lower than $15 \mathrm{~mL} / \mathrm{min} / 1.73 \mathrm{~m}^{2}$ [1]. The high risk of mortality observed in patients with ESRD is mainly due to cardiovascular events, the risks of which are 10- to 20-fold higher in these patients compared to non-CKD subjects and are significantly increased by the presence of diabetes mellitus [4,6,7]. In order to prevent cardiovascular events, patients with ESRD receive anticoagulant or antiplatelet therapy [8,9].

The benefits of low doses (75 to $100 \mathrm{mg} / \mathrm{d}$ ) of aspirin (acetylsalicylic acid or ASA), an antiplatelet agent, as prophylactic drug for some specific types of cardiovascular events (atherosclerotic and ischemic events) in CKD and patients with ESRD have been reported in several studies. For instance, the impact of low-dose aspirin $(75 \mathrm{mg} / \mathrm{d})$ versus placebo on the risk of cardiovascular events was reported in an interventional study of 3619 CKD patients with hypertension (eGFR <60 $\mathrm{mL} / \mathrm{min} / 1.73 \mathrm{~m}^{2}$ at enrollment) [10]. The hazard ratio (HR) of cardiovascular events decreased by $15 \%$ (HR $0.85,95 \%$ CI 0.61 to $1.17 ; P=.03)$ in patients with an eGFR of 45 to 59 $\mathrm{mL} / \mathrm{min} / 1.73 \mathrm{~m}^{2}$ and $66 \%$ for patients with eGFR <45 $\mathrm{mL} / \mathrm{min} / 1.73 \mathrm{~m}^{2}$ (HR $0.34,95 \%$ CI 0.17 to $0.67 ; P<.05$ ) [10]. Among secondary end points, a $36 \%$ reduction in the rate of myocardial infarction (HR $0.64,95 \%$ CI 0.39 to $1.03 ; P=.08$ ) was observed in patients with an eGFR of 45 to $59 \mathrm{~mL} / \mathrm{min} / 1.73$ $\mathrm{m}^{2}$, and subjects with eGFR $<45 \mathrm{~mL} / \mathrm{min} / 1.73 \mathrm{~m}^{2}$ had a rate reduction of $69 \%$ (HR $0.31,95 \%$ CI 0.11 to $0.85 ; P<.05)$. Stroke, cardiovascular mortality, and total mortality were also reduced by $50 \%$ to $80 \%$ in patients with eGFR $<45 \mathrm{~mL} / \mathrm{min} / 1.73$ $\mathrm{m}^{2}$ receiving aspirin compared to placebo [10]. A systematic review of 2572 randomized controlled trials, meta-analyses, and systematic reviews (27 retained) reported that aspirin was associated with a $6 \%$ reduction in the relative risk (RR) for all-cause mortality (RR $0.94,95 \%$ CI 0.88 to 1.00 ), $10 \%$ reduction in major cardiovascular events (RR $0.90,95 \%$ CI 0.85 to 0.96 ), and $15 \%$ reduction in total coronary heart disease (RR $0.85,95 \%$ CI 0.69 to 1.06 ) [11]. Last, a large case-control observational study performed on stroke patients with ESRD undergoing dialysis between 1998 and 2006 and exposed $(\mathrm{n}=763)$ or not $(\mathrm{n}=666)$ to aspirin $(80$ to $325 \mathrm{mg} / \mathrm{d})$ showed significantly lower rates of all-cause mortality (HR 0.671, 95\% CI 0.580 to $0.777 ; P<.001)$ and readmission to hospital for ischemic stroke (HR $0.715,95 \%$ CI 0.580 to $0.882 ; P=.002$ ) in the group receiving aspirin versus placebo, without any significant increase of risk of bleeding $(P=.29)$ [12].

However, there is a lack of information about the prophylactic efficacy of aspirin for all types of thrombotic events that patients with ESRD may develop. There is also a gap of knowledge concerning the safety profile of aspirin in patients with ESRD. Concerning the risk of aspirin-related bleeding, there is some discrepancy between the results of observational and interventional studies, as an increased risk of bleeding has been reported in some observational studies $[13,14]$. However, interventional studies of patients with ESRD have found that low doses of aspirin are not associated with an increased risk of major bleeding in dialysis patients, despite an apparent increased risk of minor bleeding (eg, gastrointestinal bleeding) $[10,15]$. This discrepancy highlights the fact that the mechanism of aspirin-related bleeding events is not yet fully understood. Some authors have suggested that the prophylactic efficacy of aspirin and risk of bleeding related to this drug may be influenced by a phenomenon of platelet hyperreactivity [16]. However, further research is necessary to establish the real impact of platelet hyperreactivity on aspirin's safety profile in patients with ESRD.

\section{Objectives}

The primary and secondary objectives of this study are to evaluate aspirin's prophylactic efficacy and safety in patients 
with ESRD. This will include (1) the assessment of aspirin prophylactic efficacy for all types of thrombotic events that patients with ESRD may develop, namely nonfatal stroke, nonfatal myocardial infarction, arteriovenous fistula thrombosis, and cardiac mortality, and (2) the assessment of aspirin-related major bleeding events using Thrombolysis in Myocardial Infarction (TIMI) criteria [17-19]. The study's secondary objectives are to test for effect modification of treatment by the presence of type 2 diabetes mellitus or platelet hyperreactivity and compare the rate of TIMI minor bleeding between treatment groups.

Our hypothesis is that aspirin is superior to placebo as prophylactic therapy for thrombotic events in patients with ESRD on hemodialysis without increasing the risk of major bleeding.

\section{Methods}

\section{Participants, Interventions, and Outcomes}

\section{Study Design}

We will perform a phase 2 randomized, single-center, placebo-controlled, triple-blind, superiority clinical trial with $1: 1$ allocation to receive either $100 \mathrm{mg}$ of aspirin per day or placebo by mouth for 12 months. Randomization will be stratified based on 2 baseline characteristics: (1) the presence versus absence of type 2 diabetes mellitus and (2) the presence versus absence of platelet hyperreactivity. The study will start following approval of the Institutional Review Board (IRB) and will follow the ethical principles of the Declaration of Helsinki of the World Medical Association. The study design is illustrated in Figure 1.

\section{Study Setting}

The trial involves patients on chronic intermittent hemodialysis and will be conducted in a large dialysis center (ie, with $\geq 2000$ patients on RRT).

\section{Eligibility Criteria}

The study population consists of men or women with ESRD who are at least 18 years of age and have started chronic intermittent hemodialysis in the previous 3 months.

Exclusion criteria include any contraindications to ASA, concurrent treatment with anticoagulants or platelet aggregation inhibitors, and pregnancy or lactation. Patients with life-threatening conditions other than renal or vascular disease will also be excluded from the trial: all types of cancer, liver disease, AIDS, or severe lung disease. ESRD due to glomerulopathy has a different pattern of mortality [20], which precludes its inclusion in this trial. Patients on other modalities of RRT will also be excluded.

\section{Interventions}

The intervention is an enteric-coated oral pill containing 100 mg of ASA, administered once daily, after lunch, over 12 months. The comparator will be a placebo tablet with the same characteristics as the active pills. Both arms will have the same administration schedule and will start treatment the day after randomization (day 0).

Figure 1. Illustration of the study design. Participants will be randomized in block sizes of 4,6 , and 8 . Stratification factors: type 2 diabetes; platelet hyperreactivity.

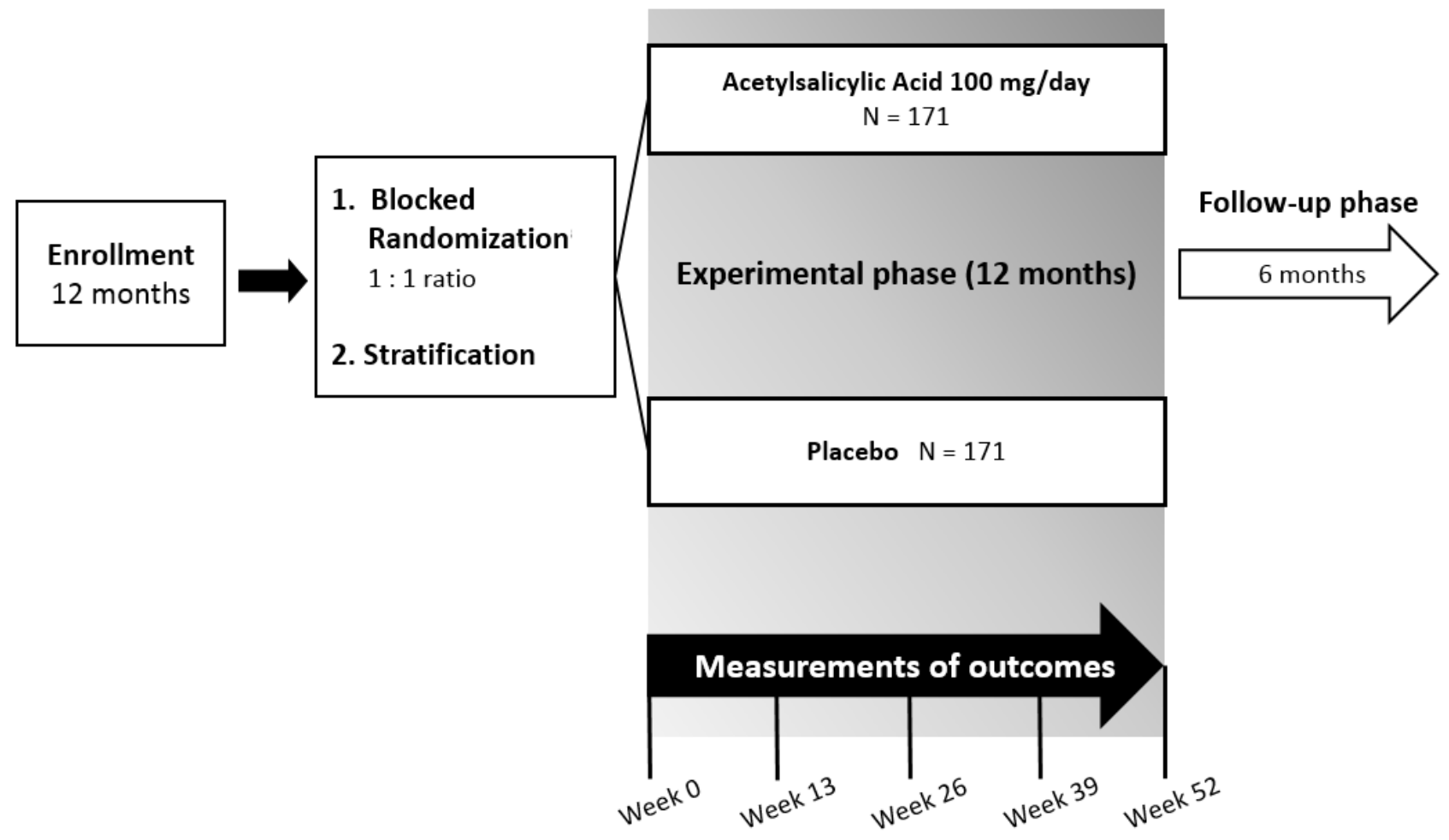




\section{Adherence}

At every hemodialysis session, participants will receive training about the importance of taking the drug as prescribed by viewing a 10-minute educational video about medication compliance on a digital tablet.

Study patients will have monthly visits with the study team to check on treatment adherence and answer any questions. During these visits, patient compliance with treatment will be assessed by direct questioning and by counting returned tablets provided on a monthly basis. Good adherence will be defined as taking at least $80 \%$ of the prescribed daily dose and attending $100 \%$ of the visits. If a patient withdraws from the trial, the study coordinator will contact the participant to find out the reasons for withdrawal.

\section{Outcomes}

The primary outcome is the incidence of a composite event including all of the following: cardiac death, nonfatal myocardial infarction, nonfatal stroke, arteriovenous fistula thrombosis, and TIMI major bleeding [19] by 12 months of treatment. The secondary outcomes are effect modification of treatment efficacy by the presence of type 2 diabetes mellitus or platelet hyperreactivity and the rate of TIMI minor bleeding between treatment groups.

According to the TIMI criteria, major bleeding is defined as the presence of any intracranial bleeding (excluding microhemorrhages $<10 \mathrm{~mm}$ evident only on gradient-echo magnetic resonance imaging), fatal bleeding (bleeding that directly results in death within 7 days), or clinically overt signs of hemorrhage associated with a drop in hemoglobin of $\geq 5 \mathrm{~g} / \mathrm{dL}$ or a $\geq 15 \%$ absolute decrease in hematocrit [19]. Minor bleeding is defined as clinically overt with a hemoglobin drop of 3 to 5 $\mathrm{g} / \mathrm{dL}$ [19].

The profile of platelet reactivity will be determined by multiple electrode aggregometry using a Multiplate analyzer (Dynabyte $\mathrm{GmBH}$ ). This method was chosen because it currently is the most efficient assay used to assess platelet reactivity in humans [21]. The Multiplate will measure platelet activity (defined as aggregation capability after activation with adenosine diphosphate) as the area under the curve reported in area units multiplied by time (AUxmin). Platelet hyperreactivity is defined based on the manufacturer's recommendations and published data, with a cutoff of 50 AU $\times$ min for adenosine diphosphate-induced aggregation [22].

\section{Outcomes Assessment}

A blinded multidisciplinary team composed of a nephrologist, cardiologist, and nurse will follow participants and review medical records and adjudicate outcomes and end points in the study.

A medical appointment will be scheduled for weeks 0, 13, 26, 39 , and 52, where the multidisciplinary team will assess the primary and secondary outcomes and report them to the Data
Coordinating Center (DCC). At each visit, a venous blood sample $(5 \mathrm{~mL})$ will be collected in order to measure hematocrit, hemoglobin, and platelet hyperreactivity.

After the end of the treatment phase of the trial, participants will be followed for an additional 6 months to assess further adverse events. For that purpose, the multidisciplinary team will contact patients monthly during their hemodialysis sessions. The total length of the study will be 30 months.

\section{Study Variables}

The outcome variables are the incidence of cardiac death, nonfatal myocardial infarction, nonfatal stroke, and arteriovenous fistula thrombosis, as well as the presence or absence of bleeding and its severity based on TIMI criteria [19]. The treatment variables are the exposure to the intervention (aspirin $100 \mathrm{mg} / \mathrm{d}$ or placebo). The stratification variables are the presence versus absence of type 2 diabetes mellitus and the presence versus absence of platelet hyperreactivity.

\section{Participant Timeline}

The schedule and procedures during patient visits to the participating center (recruitment, hemodialysis sessions, and monthly assessment of outcomes) are summarized in Table 1.

\section{Sample Size}

Sample size was calculated based on estimates for the rate of events in the primary composite outcome over 1 year: TIMI major bleeding (placebo $1 \%$ vs aspirin 2.5\% [15]), cardiovascular events (placebo 3.0\% vs aspirin 2.5\% [10]), and fistula thrombosis (placebo 19\% vs aspirin 8\% [23]), for a total 1 -year event rate of $23 \%$ in the placebo group and $13 \%$ in the aspirin group. A sample size of 318 patients (159 per arm) provides $80 \%$ power to detect a difference between groups of this magnitude (corresponding to an HR of 0.53) using a 2-sided log-rank test and alpha=.05 (Power Analysis and Sample Size Software version 14, NCSS LLC). The sample size for the trial was increased to 342 patients (171 per arm) to account for estimated attrition of $10 \%$.

\section{Recruitment}

The estimated hemodialysis admission rate at a large dialysis center is 50 patients per month. Therefore, we expect to include 8 to 10 patients every week, completing our recruitment goal in 9 months. We propose a recruitment period of 12 months in case enrollment is slower than expected.

Eligible patients will be identified using medical records, clinician invitation letters, and internal flyers posted in patient areas. The study team will approach eligible patients to invite them to participate in the trial. All aspects of the study will be explained and all participants who agree to participate must provide written informed consent. One venous blood sample will be collected prior to randomization in order to assess platelet reactivity profile, a stratification factor for randomization. 
Table 1. Schedule and procedures during patient visits to the participating center.

\begin{tabular}{|c|c|c|c|c|c|c|}
\hline \multirow[t]{2}{*}{ Procedure } & \multirow{2}{*}{$\begin{array}{l}\text { Enrollment phase ( } 12 \text { months) } \\
\text { Preliminary visit ( } 369 \text { to } 3 \text { days } \\
\text { before the experimental phase) }\end{array}$} & \multicolumn{2}{|c|}{ Experimental phase (12 months) } & \multirow[b]{2}{*}{ Every 30 days } & \multirow[b]{2}{*}{$\begin{array}{l}\text { Weeks } 13, \\
26,39 \text { and } \\
52\end{array}$} & \multirow{2}{*}{$\begin{array}{l}\text { Follow-up (6 months) } \\
\text { Hemodialysis sessions } \\
\text { (every } 30 \text { days) }\end{array}$} \\
\hline & & $\begin{array}{l}\text { First medical } \\
\text { visit (day 0) }\end{array}$ & $\begin{array}{l}\text { Hemodialysis ses- } \\
\text { sions (approximate- } \\
\text { ly every } 3 \text { days) }\end{array}$ & & & \\
\hline Informed consent form & $X^{a}$ & & & & & \\
\hline $\begin{array}{l}\text { Physical exam/medical } \\
\text { history and medication } \\
\text { review }\end{array}$ & $\mathrm{X}$ & & & & & \\
\hline Inclusion/Exclusion & $\mathrm{X}$ & & & & & \\
\hline $\begin{array}{l}\text { Collection of a blood } \\
\text { sample }^{b}\end{array}$ & & $\mathrm{X}$ & & & $\mathrm{X}$ & \\
\hline Randomization & & $\mathrm{X}$ & & & & \\
\hline $\begin{array}{l}\text { Patients' meeting with } \\
\text { the study team and oral } \\
\text { training about compli- } \\
\text { ance to treatment }\end{array}$ & & $X$ & & $\mathrm{X}$ & & \\
\hline $\begin{array}{l}\text { Provision of the neces- } \\
\text { sary number of pills (on } \\
\text { a monthly basis) }\end{array}$ & & $\mathrm{X}$ & & $\mathrm{X}$ & & \\
\hline $\begin{array}{l}\text { Patients' training focused } \\
\text { on compliance (short ed- } \\
\text { ucative video) }\end{array}$ & & & $\mathrm{X}$ & & & \\
\hline $\begin{array}{l}\text { Counting of returned } \\
\text { pills }\end{array}$ & & & & $\mathrm{X}$ & & \\
\hline $\begin{array}{l}\text { Blinding assessment } \\
\text { (questionnaire) }\end{array}$ & & & & $X$ & & \\
\hline $\begin{array}{l}\text { Measurement of primary } \\
\text { and secondary outcomes } \\
\text { during consultations with } \\
\text { a nephrologist and a car- } \\
\text { diologist }\end{array}$ & & $\mathrm{X}$ & & & $\mathrm{X}$ & \\
\hline $\begin{array}{l}\text { Assessment of later } \\
\text { bleeding adverse events }\end{array}$ & & & & & & $X$ \\
\hline
\end{tabular}

${ }^{\mathrm{a}} \mathrm{X}$ indicates the time at which each procedure will occur.

${ }^{\mathrm{b}}$ Assessment of the hematocrit, hemoglobin concentration, and platelet hyperreactivity profile.

${ }^{\mathrm{c}}$ Number of events for cardiac death, nonfatal myocardial infarction, nonfatal stroke, arteriovenous fistula thrombosis, and assessment of major and minor bleeding using the Thrombolysis in Myocardial Infarction scale.

\section{Assignment of Interventions}

\section{Sequence Generation}

Patients will be allocated to 1 of the 2 study groups based on a computed-generated blocked randomization, with stratification by 2 factors (type 2 diabetes mellitus and platelet hyperreactivity). For that purpose, randomly permuted blocks of sizes 4,6 , and 8 will be used in order to maintain the integrity of the randomization and blinding [24]. The software used to generate the sequence is available at randomization.com, and the study pharmacists will coordinate the randomization, treatment assignment, and delivery of study medication [25].

The research pharmacists will determine the study allocation and randomization and will be the only individuals to know the identity of the drugs delivered.

\section{Blinding}

The trial is triple-blinded to the treatment allocation: participants, study clinicians, and staff, as well as data analysts, will not know participant treatment assignments. Blinding will be assured by the use of a placebo comparator, which will be identical in look and taste to the active drug and will last until the data are analyzed.

A questionnaire will be used to assess the effectiveness of the participant's blinding. This will be performed according to the methodology proposed by Rees and collaborators [26]. Every 2 weeks, participants will be asked to complete a survey about the intervention they think have received (active intervention, placebo, or unknown) and their level of certainty on a Likert scale. The accuracy of their answers will be evaluated using the Howard index, and differences in beliefs between 2 successive questionnaires will be assessed using the Fisher exact test. 
Participants' beliefs about their treatment assignment are considered consistent if all responses are sequentially identical over time, except one change of opinion that may be explained by a lack of blinding [26].

\section{Emergency Unblinding}

In exceptional circumstances, unblinding may happen if knowledge of the actual treatment is essential for further management of the patient. In case of severe adverse events, investigators will discuss unblinding within 24 hours with a medical advisor from the Data and Safety Monitoring Board who is not involved with the trial. Unblinding will take place by the research pharmacy immediately after the decision is made.

\section{Data Collection, Management Analysis, and Monitoring}

\section{Data Collection}

We intend to minimize missing data by having monthly visits with patients. An adherence check will be done by counting returned tablets as previously stated.

Regarding data collection and storage, the study team will be trained to oversee all key aspects of the protocol: (1) methodology, forms, and tools that must be used for the collection, entry, monitoring, and editing of data; (2) appropriated methods to communicate among investigators and between investigators and participants; and (3) importance of reporting data as close to real time as possible during the course of the study.

\section{Data Management}

For quality control, patient records (source documents) will be stored at the site, and the original data will be shared with the DCC. It is the responsibility of the investigator to keep, maintain, and provide the documents audited by IRB, sponsor, National Institutes of Health, US Food and Drug Administration, or other local regulatory agencies when necessary.

After the collection of study data, patient identification will be encoded, and only the investigator will have access to this information, in accordance with the Good Clinical Practices and the Declaration of Helsinki regarding confidentiality [27].

The collected data will be entered electronically in a Research Electronic Data Capture management system. This database is a cloud-based system, and it will have a backup in a hard disk in the DCC. The dataset will be encrypted in order to guarantee data safety and confidentiality.

\section{Statistical Methods}

Statistical analysis will be performed including all randomized patients according to their assigned treatment group (ie, intention-to-treat). The software used will be Stata 14 (StataCorp LLC). All testing will be 2 -sided with statistical significance defined as $P<.05$. The primary composite outcome (first time-to-event of thrombotic events and TIMI major bleeding) will be analyzed with Kaplan-Meier curves and a log-rank test to detect difference between the groups. Cox proportional hazards regression may be used to adjust for relevant covariates, if appropriate (eg, for any unbalanced baseline characteristics that may occur by chance). Similarly, secondary outcomes will be analyzed with Kaplan-Meier curves and log-rank tests for all events. For the secondary objectives, interaction terms will be included in the Cox models above to test for interaction between treatment status and (1) the presence of type 2 diabetes and (2) platelet hyperreactivity. The proportion of patients with TIMI minor bleeding in each group will be compared with a chi-square test. If necessary, multiple imputation will be used for missing data.

\section{Data Monitoring}

A Data and Safety Monitoring Board consisting of an independent nephrologist and cardiologist (adverse events may happen mainly in those fields) and statistician is planned to oversee the trial. Based on federal regulations, an ethicist may be included. According to our inclusion and exclusion criteria, no vulnerable population is targeted.

\section{Ethics and Dissemination}

\section{Institutional Review Board Submission}

Prior to recruitment of study subjects, the full study protocol will be submitted to the local research ethics committee and IRB for evaluation and approval.

\section{Registration}

The trial will be registered at ClinicalTrials.gov.

\section{Results}

This is a protocol for a randomized clinical trial. It must be submitted to an ethical committee and registered at ClinicalTrials.gov before we can specify dates of data collection or the beginning of the study. These practical aspects also depend on the decisions of the center where the trial would be performed.

\section{Discussion}

\section{Summary}

Finding a safe preventive measure for thrombotic events for patients with ESRD on hemodialysis is of utmost importance, as it would reduce the number of fistula thrombosis and cardiovascular events and have a direct impact on morbidity and mortality rates. This clinical trial will provide data on the safety of antiplatelet blockade with aspirin, which is a possible preventive measure for thrombosis, and assess the impact of the intervention in hemodialysis patients. In addition, it will yield essential information to foster further interventional trials and may help revise international guidelines for the prevention of thrombotic disease in patients with ESRD on hemodialysis.

\section{Strengths and Limitations}

The main strength of the trial is its study design, which includes allocation concealment, randomization, and triple-blinding in order to reduce possible bias. In addition, stratification will balance 2 variables that are strongly associated with the outcome, type 2 diabetes mellitus and platelet hyperreactivity, and will allow us to explore whether patients with these 
characteristics differentially respond to aspirin therapy. Overall, the study protocol is feasible for both investigators and patients, as study enrollment and subsequent visits will take place during or following the patients' standard hemodialysis sessions.

Potential limitations of the study protocol include the difficulty in interpreting a composite outcome. The use of a composite primary outcome is supported by the fact that major bleeding rates are very rare, requiring a large sample size and lengthy study duration that would render the trial unfeasible. We address this issue by adding efficacy outcomes related to thrombosis to major bleeding outcomes. In order to clarify interpretation of the primary composite outcome, they are individualized in the secondary analysis. Regarding the study population, peritoneal dialysis patients will not be included as most do not have arteriovenous fistula and, hence, are not at risk for fistula thrombosis. Furthermore, glomerular disease patients who developed ESRD will not be included, as they show a different pattern of morbidity and mortality [20]. Another limitation is the possibility that we do not meet the planned recruitment time, as it will affect study power and validity. For that matter, we allow 12 more weeks for recruitment than initially planned. Last, despite the simplicity of drug administration, adherence is always a potential problem, which will be dealt with by identification of nonadherent patients and systematic training.

\section{Conclusion}

The study protocol will provide essential evidence to foster further clinical research on preventive measures of thrombotic events in hemodialysis patients. Future research is needed to provide information about the impact of preventive antiplatelet blockage on mortality and thrombotic events in these patients. Moreover, the study of potential biomarkers to identify patients who would benefit the most from the intervention is also required and may have a direct effect on drug prescription and control of adverse events. Therefore, identifying the potential safety and effectiveness of aspirin will improve morbidity and mortality, lowering the burden of such a severe disease for these patients and giving them a chance for a better and longer life.

\section{Acknowledgments}

We would like to thank our colleagues who contributed to the early development of this project: Juan Carlos Aldana, Rafael Barreto Silva, Daniella Caputo Dorta, Marcelo Costa, Caio de Assis Moura Tavares, Luís Gustavo de Mil-Homens e Vinagre, Maria Analayi Estudillo, Dayani Galato, Dolores Gonzales Fabra, Eman Hassan Satti Elsayed, Lorna Marisalva Galleguilos, Bruno Silva, Carina Vorisek, Yiling Yang, and Chen Xhi Zian.

We would also like to thank Professor Felipe Fregni (Harvard TH Chan School of Public Health and Harvard Medical School [Laboratory of Neuromodulation and Center for Clinical Research Learning, Department of Physical Medicine and Rehabilitation, Spaulding Rehabilitation Hospital]) and Professor Ben Min-Woo Illigens (Beth Israel Deaconess Medical Center and Harvard Medical School), Karla Mëtte Waldrich Tauil, Ana Rita Simoes Martins, and Christian Acosta Villegas for their support and valuable suggestions on the study design. This project received no specific grant from any funding agency in the public, commercial, or not-for-profit sectors. NMV is a scientific collaborator from the Belgian Scientific Institute of Public Health and is supported by the Belgian National Institute for Health and Disability Insurance (grant W4043.0100.8).

\section{Authors' Contributions}

AFG, CKPF, JLB, NMV, and TLC wrote the manuscript. AFG, CKPF, DAS, EEB, JLB, NMV, PGM, RW, TLC, and WEO developed the study protocol. All authors approved the final version of the manuscript.

\section{Conflicts of Interest}

None declared.

\section{References}

1. National Kidney Foundation. KDOQI clinical practice guidelines for chronic kidney disease: evaluation, classification, and stratification. Am J Kidney Dis 2002 Feb;39(2 Suppl 1):S1-S266. [Medline: 11904577]

2. Ojo A. Addressing the global burden of chronic kidney disease through clinical and translational research. Trans Am Clin Climatol Assoc 2014;125:229-243 [FREE Full text] [Medline: 25125737]

3. McClellan WM, Flanders WD. Risk factors for progressive chronic kidney disease. J Am Soc Nephrol 2003 Jul;14(7 Suppl 2):S65-S70 [FREE Full text] [Medline: 12819305]

4. Rostand SG. Coronary heart disease in chronic renal insufficiency: some management considerations. J Am Soc Nephrol 2000;11(10):1948-1956.

5. Neild GH. Life expectancy with chronic kidney disease: an educational review. Pediatr Nephrol 2017 Dec;32(2):243-248 [FREE Full text] [doi: 10.1007/s00467-016-3383-8] [Medline: 27115888]

6. Bhatti NK, Karimi GK, Paz Y, Nazif T, Moses JW, Leon MB, et al. Diagnosis and management of cardiovascular disease in advanced and end-stage renal disease. J Am Heart Assoc 2016 Dec 04;5(8) [FREE Full text] [doi: 10.1161/JAHA.116.003648] [Medline: 27491836]

7. Quarles LD. Reducing cardiovascular mortality in chronic kidney disease: something borrowed, something new. J Clin Invest 2013 Feb;123(2):542-543 [FREE Full text] [doi: 10.1172/JCI67203] [Medline: 23298840] 
8. Vlachopanos G, Ghalli FG. Antithrombotic medications in dialysis patients: a double-edged sword. J Evid Based Med 2017 Feb;10(1):53-60. [doi: 10.1111/jebm.12235] [Medline: 28276631]

9. Dastani M. Coronary artery disease in patients with chronic kidney disease: a brief literature review. Rev Clin Med 2015;2(4):182-186. [doi: 10.17463/RCM.2015.04.005]

10. Jardine MJ, Ninomiya T, Perkovic V, Cass A, Turnbull F, Gallagher MP, et al. Aspirin is beneficial in hypertensive patients with chronic kidney disease: a post-hoc subgroup analysis of a randomized controlled trial. J Am Coll Cardiol 2010 Sep 14;56(12):956-965 [FREE Full text] [doi: 10.1016/j.jacc.2010.02.068] [Medline: 20828648]

11. Sutcliffe P, Connock M, Gurung T, Freeman K, Johnson S, Kandala N, et al. Aspirin for prophylactic use in the primary prevention of cardiovascular disease and cancer: a systematic review and overview of reviews. Health Technol Assess 2013 Sep;17(43):1-253 [FREE Full text] [doi: 10.3310/hta17430] [Medline: 24074752]

12. Chen C, Lee K, Lee CT, Lai W, Huang Y. Effectiveness and safety of antiplatelet in stroke patients with end-stage renal disease undergoing dialysis. Int J Stroke 2014 Jul;9(5):580-590. [doi: 10.1111/ijs.12254] [Medline: 24597578]

13. Kim AJ, Lim HJ, Ro H, Ko K, Han SY, Chang JH, et al. Low-dose aspirin for prevention of cardiovascular disease in patients with chronic kidney disease. PLoS One 2014;9(8):e104179 [FREE Full text] [doi: 10.1371/journal.pone.0104179] [Medline: 25093403]

14. Olesen JB, Lip GYH, Kamper A, Hommel K, Køber L, Lane DA, et al. Stroke and bleeding in atrial fibrillation with chronic kidney disease. N Engl J Med 2012 Aug 16;367(7):625-635. [doi: 10.1056/NEJMoa1105594] [Medline: 22894575]

15. Baigent C, Landray M, Leaper C, Altmann P, Armitage J, Baxter A, et al. First United Kingdom Heart and Renal Protection (UK-HARP-I) study: biochemical efficacy and safety of simvastatin and safety of low-dose aspirin in chronic kidney disease. Am J Kidney Dis 2005 Mar;45(3):473-484. [doi: 10.1053/j.ajkd.2004.11.015] [Medline: 15754269]

16. Altheeb Z, Sbitan A, Shabiah M, Debari V, Hamdan A, Bikkina M, et al. Platelet reactivity unit in predicting risk of bleeding in patients undergoing coronary artery bypass graft surgery. Am J Ther 2016;23(6):e1537-e1541. [doi:

10.1097/MJT.0000000000000208] [Medline: 25909923]

17. Hage FG, Venkataraman R, Zoghbi GJ, Perry GJ, DeMattos AM, Iskandrian AE. The scope of coronary heart disease in patients with chronic kidney disease. J Am Coll Cardiol 2009 Jun 09;53(23):2129-2140 [FREE Full text] [doi: 10.1016/j.jacc.2009.02.047] [Medline: 19497438$]$

18. Kuo T, Tseng C, Lin W, Chao J, Wang W, Li C, et al. Association between vascular access dysfunction and subsequent major adverse cardiovascular events in patients on hemodialysis: a population-based nested case-control study. Medicine (Baltimore) 2015 Jul;94(26):e1032 [FREE Full text] [doi: 10.1097/MD.0000000000001032] [Medline: 26131808]

19. Mehran R, Rao SV, Bhatt DL, Gibson CM, Caixeta A, Eikelboom J, et al. Standardized bleeding definitions for cardiovascular clinical trials: a consensus report from the Bleeding Academic Research Consortium. Circulation 2011 Jun 14;123(23):2736-2747 [FREE Full text] [doi: 10.1161/CIRCULATIONAHA.110.009449] [Medline: 21670242]

20. Saran R, Li Y, Robinson B, Ayanian J, Balkrishnan R, Bragg-Gresham J, et al. US Renal Data System 2014 annual data report: epidemiology of kidney disease in the United States. Am J Kidney Dis 2015 Jul;66(1 Suppl 1):S1-S305. [doi: 10.1053/j.ajkd.2015.05.001] [Medline: 26111994]

21. Leunissen TC, Janssen PWA, Ten BJM, Moll FL, Korporaal SJA, de Borst GJ, et al. The use of platelet reactivity testing in patients on antiplatelet therapy for prediction of bleeding events after cardiac surgery. Vascul Pharmacol 2016 Feb;77:19-27. [doi: 10.1016/j.vph.2015.12.002] [Medline: 26724555]

22. Christ G, Siller-Matula JM, Francesconi M, Dechant C, Grohs K, Podczeck-Schweighofer A. Individualising dual antiplatelet therapy after percutaneous coronary intervention: the IDEAL-PCI registry. BMJ Open 2014 Oct 31;4(10):e005781 [FREE Full text] [doi: 10.1136/bmjopen-2014-005781] [Medline: 25361837]

23. Palmer SC, Di ML, Razavian M, Craig JC, Ravani P, Perkovic V, et al. Antiplatelet therapy to prevent hemodialysis vascular access failure: systematic review and meta-analysis. Am J Kidney Dis 2013 Jan;61(1):112-122. [doi: 10.1053/j.ajkd.2012.08.031] [Medline: 23022428]

24. Efird J. Blocked randomization with randomly selected block sizes. Int J Environ Res Public Health 2011 Jan;8(1):15-20 [FREE Full text] [doi: 10.3390/ijerph8010015] [Medline: 21318011]

25. Dallal GE. Website randomization. URL: http://www.randomization.com [accessed 2018-05-16] [WebCite Cache ID 6zSGG596Y]

26. Rees JR, Wade TJ, Levy DA, Colford JM, Hilton JF. Changes in beliefs identify unblinding in randomized controlled trials: a method to meet CONSORT guidelines. Contemp Clin Trials 2005 Feb;26(1):25-37. [doi: 10.1016/j.cct.2004.11.020] [Medline: $\underline{15837450]}$

27. Bhatt A. International Council for Harmonisation E6(R2) addendum: Challenges of implementation. Perspect Clin Res 2017;8(4):162-166 [FREE Full text] [doi: 10.4103/picr.PICR 124 17] [Medline: 29109932]

\section{Abbreviations}

ASA: acetylsalicylic acid

CKD: chronic kidney disease

DCC: Data Coordinating Center 
ESRD: end-stage renal disease

GFR: glomerular filtration rate

HR: hazard ratio

IRB: Institutional Review Board

RR: relative risk

RRT: renal replacement therapy

TIMI: Thrombolysis In Myocardial Infarction

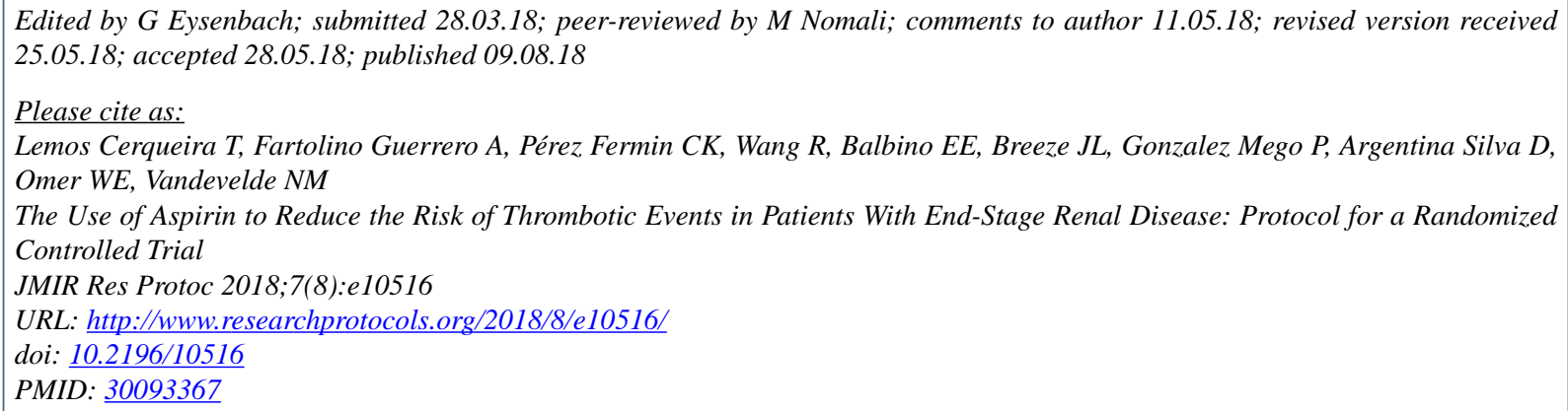

(C)Tiago Lemos Cerqueira, Armando Fartolino Guerrero, Clara Krystal Pérez Fermin, Ricardo Wang, Evelin Elfriede Balbino, Janis L Breeze, Paola Gonzalez Mego, Daniele Argentina Silva, Walid Ezzeldin Omer, Nathalie Monique Vandevelde. Originally published in JMIR Research Protocols (http://www.researchprotocols.org), 09.08.2018. This is an open-access article distributed under the terms of the Creative Commons Attribution License (https://creativecommons.org/licenses/by/4.0/), which permits unrestricted use, distribution, and reproduction in any medium, provided the original work, first published in JMIR Research Protocols, is properly cited. The complete bibliographic information, a link to the original publication on http://www.researchprotocols.org, as well as this copyright and license information must be included. 